\title{
Histopathology of Familial Versus Nonfamilial Dilated Cardiomyopathy
}

\author{
Virginia V. Michels, MD, Henry D. Tazelaar, MD, ${ }^{\dagger}$ David J. Driscoll, MD, ${ }^{\ddagger}$ \\ John C. Burnett, Jr. MD ${ }^{\S}$ Fletcher A. Miller, MD ${ }^{\S}$ A. Jamil Tajik, MD, ${ }^{\S}$ \\ Richard J. Rodeheffer, MD, ${ }^{\S}$ and Patricia P. Moll, PhD.**
}

From the ${ }^{*}$ Department of Medical Genetics and 'Pathology (H.D.T.), and Section of Cardiology, ${ }^{+}$Department of Pediatrics (D.J.D.) and \$Internal Medicine (J.C.B., F.A.M., R.J.R., and A.J.T.) Mayo Clinic, Rochester, MN; and from the **Department of Epidemiology, University of Michigan, Ann Arbor (P.P.M.).

Idiopathic dilated cardiomyopathy (DCM) is most likely a heterogenous group of diseases characterized by enlargement of one or both ventricles and ventricular dysfunction. We reported recently that the disease is familial in 20 percent of cases when first degree relatives are examined by echocardiography (1). No clinical, electrocardiographic, or echocardiographic characteristics have been identified that consistently distinguish familial from nonfamilial DCM. Because the familial nature of the disease is not necessarily apparent from review of family history alone without investigation of relatives (1), it would be helpful if histopathologic examination of right ventricular endomyocardial biopsies could provide information useful for distinguishing familial from nonfamilial disease. In one reported study, it was suggested that familial

This work was supported by grants from the National Institutes of Health (R01 H136879) and Minnesota Heart Association.

Manuscript received December 16, 1992; accepted March 10, 1993.

Address for reprints: Virginia V. Michels, MD, Department of Medical Genetics, Mayo Clinic, Rochester, MN 55905. idiopathic DCM may be identified by morphologically abnormal mitochondria evident by electron microscopy (2). However, these findings have not been confirmed, and it was unknown if these mitochondrial abnormalities were a common feature of familial disease. Therefore, we performed light and electron microscopic analysis on right ventricular endomyocardial biopsy specimens from a sequential group of 57 patients, 13 familial and 44 nonfamilial or indeterminate, with idiopathic DCM.

\section{Methods}

Biopsy specimens were identified from an unselected series of index patients with isolated idiopathic DCM who were identified because of potential enrollment in a separate, prospective study of first degree relatives to determine the proportion that had familial disease. Criteria for a diagnosis of DCM were as described previously (1). Briefly, a diagnosis of DCM was based on enlargement of one or both ventricles and impairment of left ventricular function resulting in an 

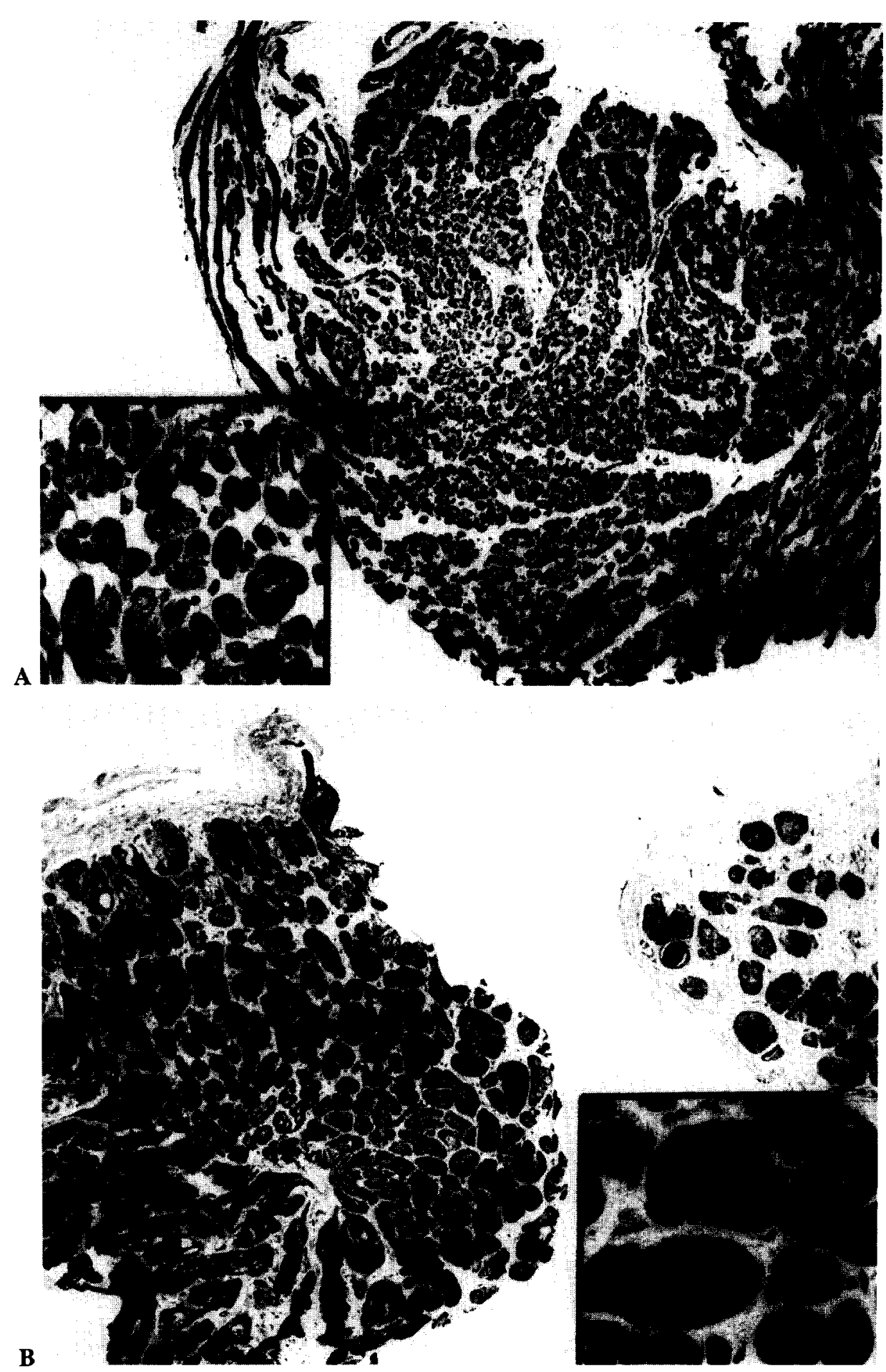

Figure 1. Photomicrograph of right ventricular endomyocardial biopsies from a 37-year-old woman with nonfamilial dilated cardiomyopathy (A) and a 42-year-old man with familial dilated cardiomyopathy (B). The biopsy in (A) shows grade 1 myocyte hypertrophy and grade 0 interstitial fibrosis and endocardial thickening. In (B) there is grade 3 myocyte hypertrophy, grade 3 interstitial fibrosis, and grade 3 endocardial thickening. The low and high power photos are at the same magnification, hematoxylin and eosin, $\times 100$; inset, $\times 400$ ). ejection fraction of $<50 \%$. Patients over 40 years of age had to have had angiographic exclusion of atherosclerotic narrowing of $\geqslant 50 \%$ of any coronary artery segment. No patient had structural cardiac lesions or other systemic disease, such as insulin dependent diabetes mellitus or cancer. No patient had known ophthalmoplegia, skeletal myopathy, lactic acidosis, or retinal pigment abnormalities suggestive of specific multisystem mitochondrial syndromes. Patients were categorized as familial, nonfamilial, or indeterminate after investigation of first degree, and sometimes second degree, relatives by physical examination, electrocardiogram, and echocardiogram. When relatives were unavailable for examination or a relative had echocardiographic findings compatible with DCM but did not meet diagnostic criteria, the family was considered indeterminate. The criteria used to diagnose relatives were the same as those described above for the index patients. Patients 
Table 1. Characteristics of Familial, Nonfamilial, and Indeterminate Dilated Cardiomyopathy Patients*

\begin{tabular}{lrcrc}
\hline & \multicolumn{3}{c}{ Patient Groups } \\
\cline { 2 - 4 } \multicolumn{1}{c}{ Characteristic } & Familial & Nonfamilial & Indeterminate & P Value \\
\hline Number (Males) & $13(3)$ & $13(8)$ & $31(16)$ & 0.11 \\
Height (cm) & $167.3 \pm 12.0$ & $161.4 \pm 34.2$ & $170.8 \pm 9.2$ & 0.47 \\
Weight (kg) & $68.9 \pm 20.5$ & $84.1 \pm 33.8$ & $74.9 \pm 18.8$ & 0.30 \\
Age at biopsy (years) & $46.7 \pm 16.1$ & $38.1 \pm 17.8$ & $43.7 \pm 15.4$ & 0.39 \\
Heart Rate (beats per minute) & $84.0 \pm 15.9$ & $83.7 \pm 16.2$ & $90.0 \pm 18.9$ & 0.52 \\
Blood pressure (mm Hg) & & & & \\
$\quad$ Systolic & $110.5 \pm 13.3$ & $122.0 \pm 18.8$ & $124.0 \pm 14.4$ & 0.06 \\
$\quad$ Diastolic & $72.1 \pm 9.6$ & $78.9 \pm 12.0$ & $75.9 \pm 15.4$ & 0.41 \\
Left ventricular size (mm) & & & & \\
$\quad$ Systole & $56.0 \pm 10.9$ & $61.3 \pm 18.7$ & $54.3 \pm 8.9$ & 0.36 \\
$\quad$ Diastole & $65.9 \pm 9.2$ & $71.2 \pm 16.7$ & $64.2 \pm 7.2$ & 0.27 \\
Ejection fraction (\%) & $32.2 \pm 11.5$ & $31.2 \pm 12.5$ & $32.2 \pm 9.7$ & 0.96 \\
\hline
\end{tabular}

*Plus-minus values are means $\pm \mathrm{SD}$.

were considered to have familial disease if one or more relatives had DCM as described previously (1).

Overall, $74 \%$ of these patients had cardiac biopsies, and all had histologic features compatible with a diagnosis of DCM, since patients with evidence of myocarditis or other specific diagnostic abnormality would have been excluded from the study.

Right ventricular endomyocardial biopsies had been performed on 57 patients, 30 females and 27 males. The mean age at biopsy was 43.1 years (range 0.5 year to 71 years). Of the patients, 13 had familial disease (based on examination of their relatives); 12 had at least one first-degree relative affected, and 1 patient had 1 affected second-degree relative and first-degree relative who was probably affected. Thirteen did not have familial disease, based on examination of their relatives. The remaining 31 patients were considered to be of indeterminate status. Eighteen of the patients considered to be indeterminate had relatives examined. Although none of the relatives met definite criteria for a diagnosis of DCM, at least 1 relative had echocardiographic abnormalities consistent with DCM and was over the age of 40 years and had not had angiography. The other 13 patients considered to be indeterminate did not have any relatives examined.

Right ventricular endomyocardial biopsies were obtained in a standard fashion. Tissue was processed for routine light microscopy and stained with hematoxylin eosin, sulphated alcian blue (for amyloid) and Prussian blue (for iron) and examined by one pathologist (H.D.T.). The mean number of pieces of myocardium evaluated for each patient was 5.3 (range 1-9). The following characteristics of the biopsy were evaluated and semiquantitatively graded $(0-3+)$ without knowledge of whether there was a positive family history: degree of myocyte hypertrophy, interstitial fibrosis, endocardial thickening, and presence of interstitial adi- pose tissue (Fig. 1). Tissue, which had been fixed in Trump's solution ( $4 \%$ formaldehyde and $1 \%$ gluteraldehyde), was processed for electron microscopy in 21 cases, including 6 familial, 7 nonfamilial, and 8 indeterminate cases. Tissue was examined using a Phillips CM-12 transmission electron microscope. Particular attention was paid to the morphology of the mitochondria. Analysis of variance was used to test for differences among the 3 groups (familial, nonfamilial, and indeterminate) of probands for quantitative traits. Nonparametric methods also were used. Since the inferences were the same, only the findings from analysis of variance are presented. Pearson's chi-square was used to compare the relative frequency of gender and biopsy characteristics among the proband groups. $P$ values less than 0.05 were considered to indicate statistical significance.

\section{Results}

The characteristics of the 3 groups of probands are presented in Table 1 . The 3 groups do not differ in the proportion of males, height, weight, age at biopsy, heart rate, blood pressure levels, left ventricular size in systole or diastole, or ejection fraction. These findings are consistent with our earlier work comparing familial patients to nonfamilial patients (1). The findings from light microscopic examination are presented in Table 2. There are no differences between the 3 groups of patients for any of the characteristics of the biopsy. When the group of familial patients are compared to the other two groups combined, there are also no differences in any characteristic. One patient with familial DCM had sufficient adipose tissue in her right ventricular biopsy to suggest a diagnosis of arrhythmogenic right ventricular dysplasia, while five categorized as nonfamilial or indeterminate DCM had similar features. 
Table 2. Summary of Light Microscopic Pathology in Familial, Nonfamilial, and Indeterminate Dilated Cardiomyopathy Patients*

\begin{tabular}{lcccc}
\hline & \multicolumn{3}{c}{ Patient Groups } \\
\cline { 2 - 5 } & Familial & Nonfamilial & Indeterminate \\
\hline Number of cases & 13 & 13 & 31 & P Value' $^{1}$ \\
$\quad$ Characteristic & & & & 0.76 \\
Pieces of myocardium examined & $4.69 \pm 1.93$ & $5.69 \pm 1.97$ & $5.42 \pm 1.96$ & 0.41 \\
Myocyte hypertrophy & $1.77 \pm 0.73$ & $1.77 \pm 0.73$ & $1.71 \pm 0.86$ & 0.22 \\
Interstitial fibrosis & $1.69 \pm 0.95$ & $1.77 \pm 1.30$ & $1.20 \pm 0.99$ & 0.80 \\
Interstitial fat & $0.92 \pm 1.19$ & $1.00 \pm 1.15$ & $0.80 \pm 0.96$ & 0.29 \\
Endocardial Thickening & $0.27 \pm 0.90$ & $0.73 \pm 1.19$ & $\mathbf{0 . 3 3} \pm 0.82$ & \\
\hline
\end{tabular}

*Plus-minus values are means $\pm \mathrm{SD}$.

${ }^{1} p$ value based on relative frequencies of graded $(0-3+)$ characteristic.

${ }^{2}$ Based on 11 familial, 11 nonfamilial and 24 indeterminate patients with evaluable endocardium.

Examination by electron microscopy in all groups supported the light microscopic findings of hypertrophy and fibrosis. Many of the myocytes showed loss of myofibrils. Although occasional small elongated mitochondria were observed in biopsies from all groups, most mitochondria were normal in appearance (Figs. 2 and 3 ). No abnormally large mitochondria with circular arrays or parallel stacks of cristae were observed.

\section{Discussion}

The etiology of most cases of DCM is unknown. Some patients ( 6 to $8 \%$ ) have been recognized as having familial disease on the basis of a positive family history $(3,4)$. However, many patients do not have an obvious family history and are recognized as familial only after echocardiographic investigation of relatives. When

Figure 2. Electron photomicrograph, obtained by right ventricular endomyocardial biopsy, of cardiac myocyte mitochondria from a 20-year-old man with nonfamilial idiopathic dilated cardiomyopathy. The mitrochondria are normal in appearance $(\times 10,000)$.

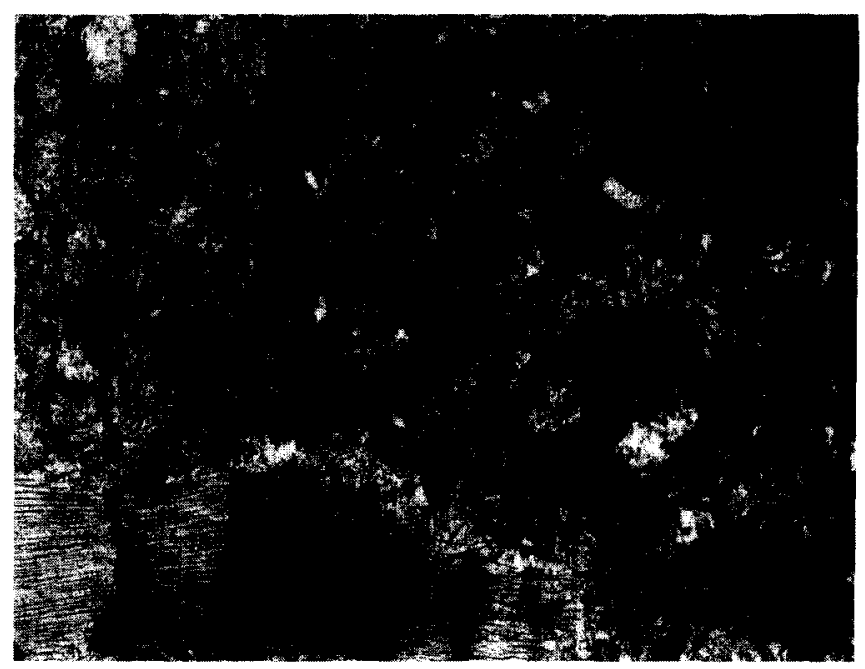

routine investigation of asymptomatic and symptomatic first degree relatives is performed, $20 \%$ of patients are shown to have familial disease (1). Because of the inconvenience and expense of studying first-degree relatives of all patients with DCM, it would be helpful to identify additional indicators of familial disease.

The histopathologic changes that generally are observed in biopsy specimens from patients with DCM are nonspecific and include interstitial fibrosis, myocyte hypertrophy, myofibrillar loss, and large and irregularly shaped hyperchromatic nuclei (5). An increased number of mitochondria of variable shape and size (abnormally small or large) with morphologic changes associated with mitochondrial degeneration are often described in DCM $(6,7,8)$. The morphology of the mitochondrial cristae and density of the matrix also may

Figure 3. Electron photomicrograph, obtained by right ventricular endomyocardial biopsy, of cardiac myocyte mitrochondria from a 37-year-old woman with familial idiopathic dilated cardiomyopathy. The mitrochondria appear normal $(\times 10,000)$.

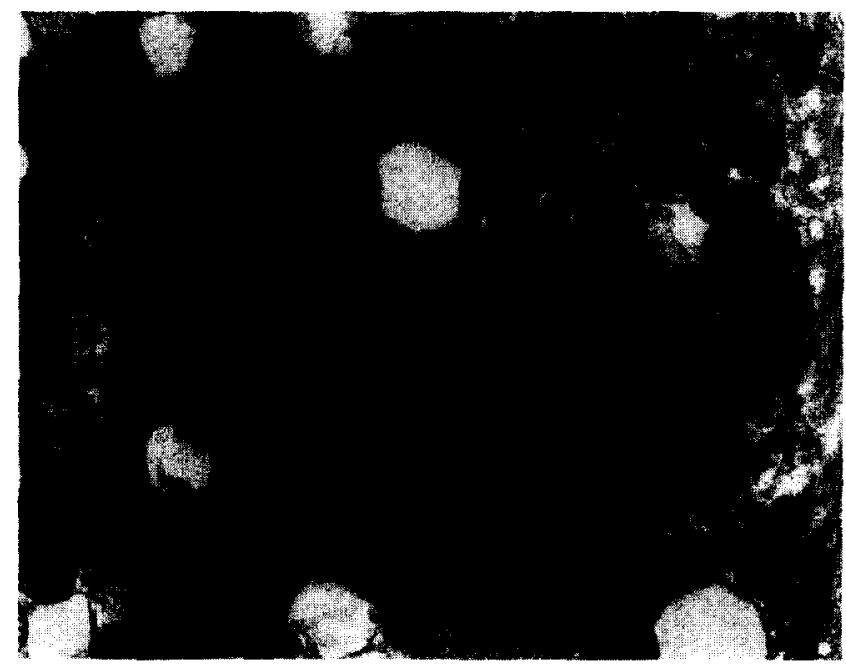


be variable $(9,10)$. Furthermore, these abnormalities may be present in only some cells from a specific heart when explanted hearts are examined (7), suggesting that sampling error may occur when biopsy specimens are analyzed.

Idiopathic dilated cardiomyopathy is a heterogenous group of diseases. Some patients have isolated cardiac disease, while others have skeletal muscle involvement with or without ophthalmologic, central nervous system, or other abnormalities. Patients with multisystem disease may have either hereditary or sporadic conditions. A subset of patients, for example those with Kearnes-Sayre syndrome, have ragged red fibers evident by skeletal muscle biopsy, indicative of mitochondrial disease. Many of these patients have been demonstrated to have mitochondrial DNA deletions, which are usually sporadic and possibly acquired. $\mathrm{Pa}$ tients with multisystem disease, including dilated cardiomyopathy, have been described consistent with a pattern of mitochondrial inheritance (i.e., only maternal transmission of the disease). Many would consider these groups of patients under a separate designation rather than referring to them as having "idiopathic" dilated cardiomyopathy. We did not include patients with multisystem disease in our study.

It has been suggested that characteristic mitochondrial changes, as demonstrated by electron microscopy, may be useful for identifying familial DCM (2). Some reported patients with mitochondrial abnormalities were infants (11) or patients also known to have skeletal myopathy, as summarized by Urie and Billingham (2). However, other patients with familial DCM and distinctive mitochondrial abnormalities had no apparent skeletal or other system involvement (2). The mitochondrial changes that have been described in familial idiopathic DCM include enlarged mitochondria with circular arrays of cristae arranged around the periphery or uniformly stacked cristae at the center of the mitochondria. In one study, these changes were described in five sets of affected siblings but were not observed in 62 age-matched controls with "congestive" cardiomyopathy or in three other familial cases of DCM. It was suggested that familial DCM might be identified by these morphologic changes of the mitochondria in some cases, but the authors recognized that additional studies were needed (2).

In our study, patients were classified as having familial, nonfamilial, or indeterminate disease in the most rigorous manner possible at this time. All our patients had idiopathic disease, without clinical or biochemical evidence of multisystem mitochondrial disease. There were no histologic or electron microscopic features of the endomyocardial biopsy specimens that allowed distinction of familial from nonfamilial or indeterminate cases. There are several possible reasons for these find- ings. First, the observations in these cases were made semiquantitatively. Morphometric measurements might be able to detect differences not discernable by our methods. Second, it is important to note that the mean age of our patients (43.4 years) was significantly greater than that of the patients described (16 years) by Urie and Billingham (2). Third, all of the patients studied by Billingham had end-stage DCM and had presented for transplant. Finally, idiopathic DCM is most likely a heterogenous disease. Although there may be a subset of patients with familial DCM who can be identified by ultrastructural mitochondrial abnormalities, in our sample of unselected, primarily adult patients, the distinction between familial and nonfamilial cases could not be made by histologic examination. Similar studies on a larger group of patients with DCM would be of interest.

The authors wish to thank study coordinator Jean L. Poellinger; Margaret Billingham, MD for helpful comments; Paul Murtaugh, PhD and Susan Gossman for technical support; and the anonymous reviewers for helpful suggestions.

\section{References}

1. Michels VV, Moll PP, Miller FA, et al. Frequency of familial dilated cardiomyopathy in a series of patients with idiopathic dilated cardiomyopathy. N Engl J Med 1992;326:77-82.

2. Urie PM, Billingham ME. Ultrastructural features of familial cardiomyopathy. Am J Cardiol 1988;62:325-327.

3. Michels VV, Driscoll DJ, Miller FA. Familial aggregation of idiopathic dilated cardiomyopathy. Am J Cardiol 1985;55:12321233.

4. Valantine HA, Hunt SA, Fowler MB, Billingham ME, Schroeder JS. Frequency of familial nature of dilated cardiomyopathy and usefulness of cardiac transplantation in this subset. Am J Cardiol 1989:63:959-963.

5. Manolio TA, Baughman KL, Rodeheffer R, et al. Prevalence and etiology of idiopathic dilated cardiomyopathy. Am J Cardiol 1992;69:1458-1466.

6. Lewis AB, Neustein HB, Takahashi M, Lurie PR. Findings on endomyocardial biopsy in infants and children with dilated cardiomyopathy. Am J Cardiol 1985;55:143-145.

7. Schaper J, Froede R, St. Hein, et al. Impairment of the myocardial ultrastructure and changes of the cytoskeleton in dilated cardiomyopathy. Circulation 1991:83:504-514

8. Rodeheffer RJ, Gersh BJ, Kennel AJ, Myocarditis. dilated cardiomyopathy, and specific myocardial disease. In: Giuliani ER, Fuster V, Gersh BJ, McGoon MD, McGoon DC, eds. Cardiology, Fundamentals and Practice. 2nd Ed. Mosby, St. Louis, 1991: $1792-1860$

9. Bosman C, Boldrini R, Fusilli S. Dilated cardiomyopathy in infancy: ultrastructural image analysis for diagnostic purposes. Pathol Res Cardiomyopathy Pathol 1989:10:807-816.

10. Caforio ALP, Rossi B, Risalti R, et al. Type 1 fiber abnormalities in skeletal muscle of patients with hypertrophic and dilated cardiomyopathy: evidence of subclinical myogenic myopathy. J Am Coll Cardiol 1989;14:1464-1473.

11. Neustein HB, Lurie PR, Dahms B, Takahashi M. An x-linked recessive cardiomyopathy with abnormal mitochondria. Pediatrics 1979:64:24-29. 